\title{
Bringing back Intrinsics to Enduring Things
}

\section{Persistence and temporary intrinsics}

In the mid-eighties, David Lewis developed an argument for perdurantism that has since become known as the argument from 'temporary intrinsics' (roughly, those properties that are temporary inasmuch as they are gained or lost and intrinsic inasmuch as they are had by things just in virtue of how they are, regardless of their relations with anything else). The argument has prompted an extensive and ongoing discussion, in spite of the fact that Lewis's presentation of the point is extremely laconic, taking the form of a short passage in a famous volume devoted to a presentation and defense of his realistic approach to modality ${ }^{1}$. Lewis's aim in developing the argument was to establish a close connection between the problem of persistence across time and the problem of qualitative change. The intended conclusion of the argument is that, either things persist by having different temporal parts at different times or it is a mystery how persistent things can change their intrinsic properties across time.

The structure of the argument is simple. First, a problem is described. Persistent objects have incompatible intrinsic properties at different times, for example they are bent at one time and straight at another. How can the bent thing existing at one time and the straight (i.e., non-bent) thing existing at another be one and the same? Second, no more than three possible solutions to the problem are envisaged. 1) Eternalist endurantism: intrinsic properties of persistent things are disguised relations they bear to times. Being straight and bent at different times is like being tall and short by comparison to different people. 2) Presentism: the only properties things have are those they have now. And nothing can be both bent and straight now. 3) Perdurantism: persistent things are made up of temporal parts. A thing can only be bent and straight at different times by having distinct temporal parts that have the incompatible properties. Third, two of the envisaged solutions are showed to be untenable. Eternalist endurantists turn intrinsic properties (e.g., shapes) into relations to times, which is a confusion: 'if we know what a shape is, we know that it is a property, not a relation' (Lewis 1986, p. 204). Presentists believe that no times exist except the present and so they 'reject persistence altogether', which 'goes against what we all believe' (ib. p.204). So the conclusion is: either intrinsic change is inexplicable, or perdurantism is true.

There are a number of ways the argument may turn out inconclusive. First, the list of solutions might be incomplete. For example, Lewis does not consider stagism as a possible solution to the problem; if stagism were true, persistent objects would not be sums of their temporal parts. Second, the rejected solutions might fail to have the purported unpalatable consequences. For example, it might be denied that presentists are bound to rejecting persistence altogether or that eternalist endurantists are bound to treating shapes as relations to times; if they are not so bound, in principle they are in a position to give good solutions to the problem of temporary intrinsics. Third, the purported consequences of the criticized solutions might be far from absurd, for example, one may argue that shapes are after all relations that objects bear to something else. If so, (some of) the

\footnotetext{
${ }^{1}$ The volume is (Lewis 1986). Two years later the argument was slightly developed in a short paper ( see Lewis 1988), and later on further discussed in greater detail in Lewis (2002).
} 
criticized solutions would not have the allegedly unpalatable consequences. Fourth, it might be argued that perdurantism has the same purportedly unacceptable consequences of (some of) the rival solutions. For example, perdurantism and endurantism seem to converge in assuming that persistent objects cannot have intrinsic shapes but only shape relations to times (according to the perdurantist, they cannot be simply bent but only bent-at- $t$, which is the case if and only if their $t$ part is simply bent). If this is true, perdurantism is no better placed than endurantism to give a solution to the problem of temporary intrinsics.

This gives a rough idea of how intricate the debate on the argument can be, but a considerable amount of discussion has focused on the impact of the argument on eternalist endurantism. To what extent are eternalist endurantists in a position to face the argument, and how? An eternalist endurantist may 'take tense seriously' (i.e., think that no tensed sentence can be correctly paraphrased in tenseless terms), and even assume that there is a genuinely ontological, non-perspectival asymmetry between what is in the present and what was in the past or will be in the future. ${ }^{2}$ But she believes that (i) reality includes the contents of different times ${ }^{3}$ (i.e., not all existing things exist at the same time) and (ii) the contents of distinct non-overlapping times can nonetheless overlap, i.e. one thing can be entirely contained in each of many disjoint times. So, Lewis' point against eternalist endurantism is that (i)-(ii) entail turning temporary intrinsics into disguised relations that persistent entities bear to times, which is inacceptable. Is Lewis right? Some of those who think not believe that, pace Lewis, it is not an obvious mistake to treat temporary intrinsics as 'relations to times' (they may various reasons for believing that: either what looks like a temporary intrinsic - for example, a shape - may be taken to be a relation in disguise, or what can be called a 'relation that something bears to a time' - for example, x's shape at $\mathrm{t}$ - may fail to be genuinely relational). Others believe that versions of eternalist endurantism can be devised that avoid turning temporary intrinsics into relations, which shows that assuming (i)-(ii) does not make it necessary to treat shapes as relations.

In what follows, I discuss three different versions of eternalist endurantism, each meant to avoid treating temporary intrinsics as relational. It is not clear that they succeed in what they are meant to do, nor that they are immune to other serious difficulties. Two of them, generally known as 'adverbialism' and 'SOFism', are familiar and controversial. I shall scrutinize Lewis' criticisms of them and argue that the perplexities are well founded. Then, I shall devise a further, to some extent new, version of eternalist endurantism which seems better placed, so I shall argue, to treat shapes and the like as really intrinsic. I shall conclude that eternalist endurantism has nothing to fear from Lewis' argument. Before that, however, let me briefly say why I think that Lewis is right both to assume that properties like straight-at-t are extrinsic and to believe that shapes and the other temporary intrinsics are not relational.

II. Relational, intrinsic, monadic properties

\footnotetext{
${ }^{2}$ On taking tense seriously, see Zimmermann $(2005,2006)$

${ }^{3}$ More precisely, according to the eternalist, reality includes entities existing at different times some of which are before others. Indeed, suppose that (i) modal realism is true, (ii) in each world only present objects exist, and (iii) no time in a world is the same as a time in another world. In such a case, eternalism would be false despite the fact that reality includes entities existing at different times.
} 
The very idea of a relation with a time might seem far from clear, so it might be thought that the property of being straight is after all intrinsic, despite the fact that a persistent object can be straight at one time and not straight at another. Forbes holds that the idea that the variable shapes are extrinsic "could be avoided by appropriate redefinition of "intrinsic" (Forbes 1987, 140). The notion of an intrinsic property is extremely difficult and controversial, ${ }^{4}$ but the general idea is that a property is intrinsic just in case, if something has the property, this can only depend on the way it is and on nothing else, so that an intrinsic property can be had by something just in case it is had by all its duplicates. And it might seem that, if $x$ is straight at a time $t$, this can only depend on the way $x$ itself is regardless of anything else (on how $x$ itself is $a t t$ ). Starting from this intuition, Haslanger $(1989,2003)$ recommends a temporally sensitive construal of the notion, according to which $P$ is an intrinsic property of $x$ (at a time $t$ ) iff $x$ has $P$ (at $t$ ) in virtue of the way $x$ is (at $t$ ), regardless of anything else. ${ }^{5}$ In this sense of 'intrinsic', something can be intrinsically bent (at one time) and intrinsically straight (at another): shapes are somehow relational, but nonetheless intrinsic.

Be it as it may, Lewis' worry seems to be still there. Lewis' intuition is that, if one knows what is a shape, one knows that objects have shapes simpliciter, i.e., regardless of anything else. This intuition is perhaps better captured by the idea that shapes are monadic, so that, given a property like straight, one object is enough to have a truth value. ${ }^{6}$ Suppose that the property that $x$ has at $t^{1}$ - say, bentness - is exactly the same that $x$ lacks at $t^{2}$. A monadic property can be treated extensionally as a function from objects to truth and falsity. How is it that bentness has different values (truth at $t^{1}$ and falsity at $t^{2}$ ) for the same argument? Either two different functions are both named 'bentness', or bentness is not really monadic. Perhaps what is meant is that, given an argument, the function has one value relative to $t^{1}$ and another relative to $t^{2}$. If so, however, bentness is no more monadic than brotherhood: just as one can have and lack the property of brotherhood relative to different people, one can have and lack the property of straightness relative to different times. What seems to be lost here is the intuition that shapes and the like are not relations but purely monadic properties. In Zimmerman's words: 'A property is really monadic if and only if the property plus a single individual can together constitute something that is true, simpliciter, not merely true relative to a time (or anything else)' (Zimmermann 2006, p. 423). Zimmerman calls these really monadic properties 'metaphysically monadic'.

So, eternalist endurantists fail to treat shapes as metaphysically monadic. Perhaps, however, this is exactly what should be done. ${ }^{7}$ Special relativity entails that even properties that seem to be monadic, like shape, are indeed polyadic inasmuch as they are frame-relative. Consider mass. Taking for granted relativity, there are two viable notions of mass: rest mass and relativistic mass. Unlike rest mass, relativistic mass is frame-dependent: an object may have a relativistic mass of one gram in a frame and one of forty grams in another. But even rest mass has sometimes been treated as relative, on the assumption that $x$ 's rest mass is nothing but its relativistic mass relative to its own frame of reference. The same goes for size. Maudlin considers a car and a tunnel that are exactly the same length when they are at rest relative to each other. When the car pass through the tunnel, however, it is both shorter than the tunnel inasmuch as it moves relative to it and longer than the

\footnotetext{
${ }^{4}$ See Langton and Lewis (1998), Humberstone (1996).

${ }^{5}$ See also Jackson 1994.

${ }^{6}$ If Haslanger and Jackson are right, relational properties can sometimes be intrinsics (which is the case with other relational properties like having exactly two proper parts) and, if Haslanger and Jackson are right, this is the case of shape-relations to times. But Lewis points out that the problem of change specifically concerns intrinsic monadic properties (see Lewis 2002, p. 4).

${ }^{7}$ See Eddon 2010.
} 
tunnel inasmuch as the tunnel moves relative to the car. According to Maudlin, this 'illustrates how seemingly nontemporal notions may be infected by hidden temporal aspects' (Maudlin 2002, p. 55). The same goes for shape: an object that is straight relative to a frame can be bent relative to another. Against our plain intuitions, time seems to play some role in seemingly monadic properties like mass, size and shape.

So far so good. But I do not see how the above considerations can prove that rest mass is extrinsic, being at best a relation that physical objects bear to themselves - which means that, if $\mathrm{x}$ has rest mass $\mathrm{m}$, this can only depend on $\mathrm{x}$ itself, regardless of anything else. Similarly, I do not see why Maudlin's example might not be taken as illustrating a situation in which relational lengths are produced by certain 'internal' properties of two physical systems taken in isolation and by their external spatiotemporal relations. After all, one might say that if new sections were attached to the car, its length relative to the tunnel would have been different. Similarly, one might say that something is intrinsically straight if the shortest line that connects its outermost extremities does not go out of it. Even if masses, shapes and sizes aren't had simpliciter, surely there is something underlying these properties (call it 'rest mass', 'rest shape' and 'rest size', if you like) that is intrinsically had by objects, regardless of anything else. And the problem remains of explaining how it is that these intrinsic features change across time.

So, let us assume that turning temporary intrinsics into relations to times is a mistake. Does the mistake necessarily follow from the basic assumptions of eternalist endurantism?

\section{Having-at-t a property}

Eternalist endurantists believe that the contents of a couple of non-overlapping times, $t^{1}$ and $t^{2}$, can nonetheless overlap (i.e. something can be entirely contained both in $t^{1}$ and in $t^{2}$ ). Either there are true contradictions, however, or nothing can be in the overlap of the contents of $t^{1}$ and $t^{2}$ unless it is contained in $t^{1}$ and has the same properties as something contained in $t^{2}$. So, suppose that a candle that is straight at $t^{1}$ and bent at $t^{2}$ is in the overlap of the contents of $t^{1}$ and $t^{2}$. If there are no true contradictions, it cannot have both straightness and bentness, but it can easily have both straightness-at- $t^{l}$ and bentness-at $-t^{2}$, and eternalist endurantists are inclined to think that what the persistent candle has are those temporalized properties. There can be in principle two ways of having straightness-at- $t$ ' : having the relation of being straight with a particular entity called ' $t$ ', and being straight in a certain way (' $t^{l}$-ly bent', so to say). Either there is one dyadic relation of straightness holding between persistent entities and times, or a congeries of monadic adverbially qualified straightnesses. Lewis' accusation of turning intrinsics into relations is addressed against the former variant of the relationalist approach (indeed, as far as I know the latter has never been defended by anyone). What is important now is that, whatever straightness-at- $t^{1}$ and bentness-at- $t^{2}$ might be, eternalist endurantists can most naturally explain how something can be straight at one time and bent at another by substituting them for plain straightness and plain bentness.

What is most natural, however, may not be necessary. According to Johnston (1987) and van Inwagen (1990), there is a variant of eternalist endurantism that brings plain straightness and bentness back into the picture. Roughly, the idea is that the candle can be straight at one moment but not at another by having-at-that-moment but not-at-the-other the property of being straight. What is affected by time is instantiation, that is, the way the candle has the intrinsic property of being straight, and not the intrinsic property itself. Call this approach 'adverbialism'. According to 
adverbialism, the temporal qualification 'at $t$ ' occurring in the sentence 'at $t$, the candle is straight' modifies neither the predicate nor the singular term but the copula that relates the former to the latter.

It is far from clear in what exact sense the copula is said to be temporally qualified. How has the phrase 'at $t$ ' to be understood in the sentence 'the candle has-at-t straightness'? Is it to be taken as attached to 'has' in the way in which 'at the hotel' is attached to 'arrived' in the sentence 'she arrived at the hotel' or rather in the way in which 'at mathematics' is attached to 'good' in the sentence 'she is good at mathematics'? Being good at mathematics is a kind of ability in a sense in which arriving at a particular hotel is not a kind of arriving or being brother of Sam is not a kind of brotherhood. When one says of someone that she is good at mathematics, the relational structure of the sentence is only apparent, as is evident from the possibility of saying the same thing with an equivalent non-relational sentence such as 'she is a good mathematician', or something like that. Being good at mathematics is not bearing a relation to a particular entity, like arriving in a place or being brother of someone. Accordingly, we can have two different versions of the third solution, of which only one is properly adverbial. Let me call the properly adverbial version 'strict adverbialism' and the other one 'pseudo-adverbialism'. According to strict adverbialism, there are a number of different dyadic kinds of instantiation (the $t^{1}$-instantiation, the $t^{2}$-instantiation and so on), having different extensions (the candle can instantiate straightness in the $t^{l}$ way and fail to instantiate it in the $t^{2}$ way). According to pseudo-adverbialism, there is only one kind of instantiation, but it is triadic: $n$-tuples instantiate $n$-adic properties relative to times. The sentence 'Now the candle is straight' must be read as 'The candle instantiates the monadic property of being straight relative to the present time'. A defense of strict adverbialism can be found in Johnston $(1987),{ }^{8}$ while van Inwagen (1990) defends a version of pseudo-adverbialism. ${ }^{9}$

Lewis (2002) criticizes adverbialism, but his arguments apply only to what I have labelled 'pseudo-adverbialism'. The criticism can be summarized as follows. If all that the candle can do is bear the relation having at to the moment $t$ and the property of being straight, it can only have the property of bearing a particular relation to $t$ and the property of being straight, not the property of being straight itself. Lewis asks: '[...] what does standing in some relation to straightness have to do with just plain being straight? And [adverbialism] still claims that to be shaped is to stand in relation to other things, inter alia to times. I say it still amounts to a denial that things have temporary intrinsics' (Lewis 1988, p. 66). Therefore, by claiming that it is instantiation of straightness instead of straightness itself that is relative to times, no progress can be made in explaining how something can have monadic straightness. For it is one thing to have a property, and quite another to bear some relation to it. 'If a relation stands between you and your properties' Lewis says, 'you are alienated from them' (Lewis 2002, p. 5). If one insists that having simpliciter a property has to be explained in terms of bearing a relation of having to that property, so that having simpliciter a property is no less relational than having it at a time, one has to face the dilemma of Bradley's regress (Bradley 1897, ch.3). Bradley's regress shows that if one tries to explain having simpliciter in terms of a kind of relational having, the explanation will never come to an end.

Perhaps Lewis understates his case. Not only is there no hope of bringing temporary intrinsics back into the picture by putting time-relativity in instantiation rather than in the instantiated property; what is far from clear is the very difference between putting time-relativity in

\footnotetext{
8 'Temporal qualification has to do with the ways individuals have properties. [...] Temporal qualifiers are often adverbs. Sam is presently fat. But he is $t$-ly thin' (Johnston 1987, p. 128)

${ }^{9}$ See van Inwagen 1990, pp. 249-250.
} 
instantiation and putting it in what is instantiated. One might say that, since instantiation is a threeplace relation, only couples of things (objects and times) can instantiate the property of being straight. Or else one might say that, since being straight is a two-place relation, only couples of things (objects and times) can instantiate the property of being straight. In both cases, the result seems to be the same: if only a couple of entities (an object and a time) can instantiate the property of being straight, then being straight is a two-place property, i.e. a relation between objects and times (and in no other case is it a two-place relation: what else could a two-place relation be?). Exactly the same can be said about predicates and satisfaction: assuming that the predicate 'straight' can be satisfied only by couples of entities (objects and times) amounts to assuming that 'straight' is a two place-predicate (what else could a two-place predicate be?).

Suppose we adopt the following convention: instead of saying that a $n$-place predicate is satisfied by an $n$-tuple, we will say that it is satisfied relative to the last member of the $n$-tuple by an $n^{-1}$-tuple (the original $n$-tuple minus its last member). Whenever $x$ is a brother of $y$, for instance, we will say that $x$ satisfies the one-place predicate 'brother' relative to $y$. In general, $n$-adic predicates will become $n^{-1}$-adic predicates, and satisfaction, just like instantiation, will become an $n^{+1}$-adic relation (if a predicate is monadic we shall say that it is satisfied relative to one object by the empty set). What consequences would that have for satisfaction, and what for properties? Precisely nothing substantial, I would say: it would be a mere lexical change. Time-relative instantiation is just time-relative bentness and straightness in disguise.

Lewis' arguments apply to what I have labelled 'pseudo-adverbialism'. What about strict adverbialism, the idea that the phrase 'at $t$ ' in 'having-at- $t$ ' works as an adverbial modifier, like 'at mathematic' in 'good at mathematics', rather than as a referential expression, like 'at the hotel' in 'arrived at the hotel'? Lewis' criticism of pseudo-adverbialism (i.e., that the addition to having of an extra place for time cannot but turn it into a relation) does not apply to strict adverbialism. Indeed, if the modifier 'at $t$ ' is treated as merely adverbial, there is no reason to consider having-at- $t$ as more relational than having simpliciter (suppose having is a 'non-relational tie'; admitting that there are a number of different kinds of non-relational ties would not make the ties more relational). So, one might wonder why Lewis does not seem to consider strict adverbialism as a viable possibility. Here are a couple of hypotheses. First, the inferential move from ' $a$ is $F$-ly $P$ ' to ' $a$ is $P$ ' is most naturally considered as correct. If a is $\mathrm{P}$ in some way, then a is $\mathrm{P}-$ for example, if I run quickly, I run. If this is the case and 'at $t$ ' behaves like an adverb, something that is bent at $\mathrm{t}^{1}$ and is straight at $\mathrm{t}^{2}$, is bent and straight, which is a plain contradiction. Second, we seem to have no intuitive grip on what it is to have a property in a $t$-ly way that does not presuppose a grasp of what time $t$ is, which means that the temporal adverb would have no meaning if ' $t$ ' had no reference. If so, being straight in some temporal way can naturally be thought of as consisting in being bent relative to a particular time, and nothing is left that can distinguish strict adverbialism from pseudo-adverbialism. Strict adverbialism requires a theory of adverbial qualification according to which a property can be had in certain ways without being had, and a semantics of temporal adverbs according to which the meanings of those adverbs are independent from the apparatus of reference to times. And it seems implausible that such a theory can be easily devised. 
Lowe, Haslanger and others ${ }^{10}$ believe that there is an easy way to get rid of the above difficulties without dismissing what matters in adverbialism. According to adverbialists, the time at which an object has or lacks a property (i) makes no difference to either the object or the property; (ii) makes a difference to the way in which the object has the property. According to Lowe and Haslanger (i) is true but (ii) is not: time modifies neither the object nor the property, but even less does it modify instantiation.

What time affects, according to Haslanger (1989), is the truth value of the proposition that something plainly has an intrinsic monadic property. When one says that the candle is straight at $\mathrm{t}$, one says that the proposition that the candle is straight simpliciter obtains or is true at $t$. Truth rather than instantiation is time relative. According to Lowe, 'To say that the having of a shape is related to a time is to say that the holding of a two-place relation is related to a time, not that a three-place relation is involved, one of whose relata is a time.' (Lowe 1998, p. 132). In other words, the idea is that 'a thing's being shaped itself stands in relation to times, not that a thing's being shaped is partly a matter of that thing's standing in relation to times' (Lowe 1988, p. 75). ${ }^{11}$

Both Lowe and Haslanger give a timeless reading of 'the candle has bentness', and both think that the candle's having bentness is time relative, i.e., it can only obtain or hold relative to times. But there are differences, because Haslanger (1989) articulates this idea in terms of propositions and their being true or false relative to times, which Lowe does not. Haslanger's underlying idea seems to be that a proposition can have as its content that an object has timelessly a monadic intrinsic property, so that when one says that the candle is bent at $t$, one says that one proposition (that the candle is timelessly bent) obtains at $\mathrm{t}$.

Lewis (2002) wonders how this proposition has to be understood. ${ }^{12}$ Since it is supposed to be true at some times and not at others, it is not a property of worlds, like a proposition that is true once and for all. It must be an egocentric proposition and particularly a property of times, intuitively the property of being a time at which the candle is bent. But it cannot just be the set of all times $t$ such that the candle is bent-at-t, otherwise it would be useless for the purpose of bringing intrinsic monadic bentness back into the picture. It must be a structured property, endowed with a quasisyntactic articulation, but among its components can occur neither bent at nor having-at, otherwise (again) it could be of no use for bringing back intrinsic bentness. If so, what turns out to be the case is that ' $[\ldots]$ within the anatomy of [...] the relational property just considered, we have reintroduced without explanation the very thing we were trying to explain: the notion of an enduring thing having a monadic intrinsic property at a time' (Lewis 2002, p. 12). Lewis concludes that Haslanger's proposal can make no substantive further step towards making eternalist endurantism compatible with temporary monadic properties.

There are more general reasons for thinking that treating truth instead of instantiation as relative to times can make no substantive difference. Obviously, the proposition expressed by the sentence 'the candle is straight' is true if and only if the referent of 'the candle' satisfies the predicate 'is straight', if and only if the candle has the property of being straight. Hence, if 'the candle' is temporally rigid, the proposition is true at some times but not at others just in case the candle has at some times but not at others the property of being straight. At least in the case of propositions expressed by subject-predicate sentences with temporally rigid subjects, treating truth

\footnotetext{
${ }^{10}$ See (Forbes 1987), (Myro 1986)

${ }^{11}$ In 'true at $\mathrm{t}$ ' and 'obtaining at $\mathrm{t}$ ', the phrase 'at $\mathrm{t}$ ' is ambiguous between a referential and an adverbial reading, in the same way as it was ambiguous in 'having-at-t'.

${ }^{12}$ See pp. 11-12
} 
as time-relative amounts to treating having as time-relative, that is, as a three-place relation. And, according to endurantists, singular terms like 'the candle' are temporally rigid, since they denote the same enduring thing at every time at which they denote anything at all. If endurantism is true, then, temporal relativity of truth is nothing other than temporal relativity of instantiation in disguise. But, as I argued earlier, temporal relativity of instantiation is nothing other than temporal relativity of intrinsics in disguise. If a provisional moral can be drawn, then, it is perhaps that the discussion about temporary intrinsics tends to return to its starting point. Both adverbialism and Haslanger's temporal relativity of truth do nothing substantive to advance the discussion in any way.

Haslanger has subsequently revised her own approach, giving up the view of truth as timerelative. The new approach, which Haslanger calls 'SOFism', 13 elaborates in an organic account ideas that are very close to Lowe's and Forbes' more sketchy intuitions. The role previously played by propositions being true at some times and not at others, is now played by types of states of affairs having tokens at some times but not at others. The relation between types and tokens state of affairs is an intricate matter, but at least the following can be said. A state of affairs type is something tenseless as the candle's being bent. A state of affairs type $A$ obtains at a time $t$ iff a state of affairs token $a$ occurs at $t$ such that $a$ is of type $A$ (or $a$ 'realizes' $A$ ). A state of affairs token of the type the candle's being bent occurs at $t$ iff (i) the candle exists at $t$; (ii) at $t$ the candle is bent (the biconditional holds regardless of whether it is the left-hand side that grounds the right-hand or vice versa). An assertive utterance of a subject-predicate sentence like 'the candle is bent' refers to a state of affairs token and says of it that it is of the type the candle's being bent. Therefore, assertive utterances express eternally true (false) propositions, even though states of affairs types obtain at times. The same enduring object - i.e., the candle - is wholly present in every token of the state of affairs type the candle's being bent, as well as in every token of the type the candle's being straight. In all these states of affairs, being bent (as well as being straight) is a monadic intrinsic property of the candle, not a relation that the candle bears to times. There is no contradiction in the candle's changing its shape, because the states of affairs types the candle's being bent and the candle's being straight never have tokens at the same time. Is this a successful way to incorporate intrinsic monadic properties in an eternalist endurantist theory of qualitative change?

I see fundamentally two reasons to be skeptical. First, I do not see how SOFism can explain how the straight candle in $t^{1}$ and the bent candle in $t^{2}$ can be one thing without being one straight and bent thing. Haslanger says that there is no contradiction between the states of affairs types the candle's being straight and the candle being bent, since they obtain at different times. But they obtain at different times (i.e., have tokens at different times) just in case the candle entirely exists in the overlap of the contents of those times, having simpliciter monadic straightness at the former time and monadic bentness at the latter. ${ }^{14}$ And how can the candle do that, granted there are no true contradictions? Relationalists about intrinsics can say: 'by bearing different shape-relations to different times, no contradiction!'. Adverbialists can say: ?by bearing different relations of having to incompatible monadic properties, ${ }^{15}$ no contradiction!'. But SOFists cannot say 'by being entirely present in two states of affairs tokens occurring at different times, and having straightness in one

\footnotetext{
13 'SOFism' is the acronym of 'State-Of-aFfairs-ism'. See (Haslanger 2003)

${ }^{14}$ The bentness [...] is nothing other than the property that the candle has when the type state of affairs obtains (in other words: it is the property of the candle in the token state of affairs)' (Haslanger 2003, p. 347).

${ }^{15}$ Or, according to 'pseudo-adverbialists', by bearing the same triadic relation of having to incompatible properties and different times.
} 
and bentness in the other', for this presupposes (or entails, or both) the very contradiction that should be dissolved.

The second worry directly concerns the intended fundamentality of Haslanger's ontology of states of affairs types and tokens. Is it by virtue of the candle's having bentness at $t$ that the state of affairs type the candle's being bent has a token at $t$, or the reverse? In the former case, the ontology of states of affairs might be seen as redundant and only particulars and properties (whatever they may be) might be considered as existent at a basic level. States of affairs types might be treated as couples of particulars and properties, states of affairs tokens as triples of particulars, properties and times such that the particular has the property at the time. If so, whatever SOFism can tell us in terms of states of affairs can be fairly paraphrased in terms of individuals, properties and times. And it would be extremely weird if a new solution to an old problem could become available just by changing our way of speaking. ${ }^{16}$

If, on the contrary, states of affairs are supposed to lie at the most basic and fundamental level of reality, particulars and properties might somehow be defined as invariances of resembling states of affairs (states of affairs types might be defined as invariances of states of affairs tokens; and particulars and properties as distinct kinds of invariances of states of affairs tokens). ${ }^{17}$ But SOFism, so understood, can hardly be treated as a variant of endurantism. ${ }^{18}$ States of affairs tokens occurring at different times can never be identical (even if they can be of the same type), so that there can be no such thing as identity across time. For sure, the same state of affairs type can obtain at different times (by having different tokens that exist at those times), but it cannot exist at different times per se. One might perhaps treat states of affairs types as immanent universals and allow each of them to coexist with all its tokens. Even in that case, states of affairs (both types and tokens) are immutable, i.e., they can never have different properties at different times. If a state of affairs token is supposed to have as its proper parts shorter states of affairs tokens, qualitative change can perhaps be reintroduced along perdurantist lines, since the shorter parts of a longer state of affairs token are likely to be qualitatively different from each other. But this is perdurantist change in disguise (coming into and going out of existence of qualitatively different but immutable entities) not the kind of 'genuine' change that the endurantist has in mind (diachronic qualitative difference cum numerical identity).

What moral can we draw? Does SOFism succeed in bringing back intrinsic monadic properties into a coherent endurantist picture of persistence and change? Much seems to depend on whether particulars and properties are taken to be primitive, states of affairs types and tokens being derivative, or the reverse. In both cases, intrinsics are brought back into the picture. In the former case, however, no reason is given to think that the picture is coherent (how can the candle be the common content of two states of affairs tokens having monadic bentness in one and monadic straightness in the other?), while in the latter the account can hardly be regarded as a variant of endurantism. In neither case, it seems, does it succeed in incorporating intrinsic monadic properties in a coherent endurantist theory of change.

\footnotetext{
${ }^{16}$ This would be the case even if the two 'ways of speaking' were just as fundamental, and intertranslatable without any semantic loss.

${ }^{17}$ An invariance is an equivalence class. Even times might perhaps be defined as a third kind of invariances between states of affairs tokens. If so, there would be nothing in the world, at a most fundamental and basic level, except state of affairs tokens and equivalence classes (invariances) of these tokens.

${ }^{18}$ So understood, indeed, SOFism can be hardly compatible even with what Wasserman (2006) calls 'Plato's paradigm', our standard model for the metaphysics of predication according to which objects have properties (however conceived of) and predicative utterances are true depending on what properties are had by objects.
} 


\section{Bringing back monadic intrinsics}

Both adverbialism and SOFism aim to find a sense in which the enduring candle itself can be said to have monadic bentness and straightness. But this may be more than what is strictly required in order to 'bring back' intrinsic monadic properties into the picture. According to Lewis, what is strictly required is that the candle's being straight (bent) at a time can be explained as consisting in plain straightness (bentness) of something, not necessarily the candle. The friend of endurance, Lewis says, treats the candle's shape as a disguised relation it bears to times, not as a monadic intrinsic property of it. But the friend of perdurance does the same. On her view, the candle is the sum of its temporal parts. Some of them are straight simpliciter, some are bent simpliciter. But the candle itself can be neither, it can only bear the relation straight or the relation bent to a time $t$, depending on whether its $t$-part is bent simpliciter or straight simpliciter. So, what distinguishes endurantism from perdurantism is not that the latter 'does away with shape-at-a-time relations. Rather, it is that the first has wrongly done away with shapes as intrinsic properties that can be had simpliciter' (Lewis 1988, p. 66). According to the perdurantist, '[...] your perduring self does not have bent simpliciter. But as much of you as exists at $t 1$ does' (Lewis 2002, p. 5), and it is in virtue of this that you bear the bent-at relation to $t 1$. The endurantist's mistake is that for her, on the contrary, 'nothing just has a shape simpliciter' (Lewis 1988, p.65).

According to Lewis, therefore, an account of intrinsic change gives monadic straightness and bentness their due insofar as: 1) it recognizes that something is straight (bent) simpliciter; 2) it is able to account for the candle's being bent (straight) at a time in terms of something being bent simpliciter. Insofar as an account of intrinsic change satisfies these constraints, it has nothing more to do in order to 'bring back' temporary intrinsics in the picture. Someone (not Lewis) may want to question whether this is enough. Presentists and stagists claim that, as long as it is something different from the candle itself that has monadic bentness (a proper temporal part of it, or the like), intrinsics are not really brought back into the picture (see for example Hinchliff 1996, Sider 2000). This idea does not simply follow from the assumption that shapes are monadic properties and not relations, and is highly controversial. At least the following, however, should perhaps be conceded. If straightness (bentness) is an intrinsic monadic property had by a number of things (temporal parts of candles, for example), then an account of change that gives intrinsics their due should 3) have an answer to the question 'is the changing candle itself bent simpliciter or not?'. As long as one takes seriously the idea that there is a class - the extension of the monadic property bent - to which some things belong, one can wonder whether the candle itself falls into that class, outside, both inside and outside, or elsewhere. And one should wonder about that, since the problem of change is precisely that the candle itself seems both to have and to fail to have bentness (at different times). Given all this, bringing intrinsics back into the picture requires no more than satisfying constraints 1)-3).

\section{Being straight simpliciter and bent at times}

Eternalist endurantists are likely to be fairly at ease with constraints 1) and 2). Lewis says that according to the endurantist 'nothing just has a shape simpliciter', but this is far from clear. The eternalist endurantist's ontology can embrace plenty of plainly bent and plainly straight entities. 
Spatial regions are one example. Momentary phases of events like parties, battles and journeys (in the case of eternalist endurantists that believe in events) are another example. Having all these plainly bent things in her ontology, the eternalist endurantist can easily account for the candle's being bent at time $t$ in terms of some of those things being bent simpliciter. She might say, for example, that the candle bears the relation bent-at to time $t$ just in case the spatial region that the candle occupies at $t$ is bent simpliciter (this is intuitively convincing: the shape of an object depends on the shape of the spatial region it exactly fills; and spatial regions have shapes simpliciter). Alternatively, she might say that the candle bears the relation bent-at to time $t$ just in case the $t$ phase of the candle's burning is bent simpliciter. (An eternalist endurantist who likes events is free to accept momentary event-phases in her ontology, which might play roughly the same role as temporal parts in a perdurantist ontology; she might perhaps assume that the enduring candle is constituted by a different event-phase at every time $t$ of its lifespan, even though it is entirely present at $t$; similarly for any enduring part of the candle).

What about constraint 3)? To decide where the candle is located in respect to the extension of the monadic property bent is a fly in the ointment for every account of change and persistence, not only for eternalist endurantism. Take perdurantism. For perdurantists, the candle is a worm having some slices plainly bent and some plainly straight. So far so good, but what about the worm itself? Is it straight simpliciter or not? One can take either of two lines. First option: unlike its instantaneous slices, the temporally thick candle extends in four dimensions and nothing that is thick in four dimensions can be meaningfully said to be straight simpliciter. Strange, the property of being straight can be exemplified by something regardless of whether it extends in one, two or three dimensions. And why not in four? But the temporal character of one of the dimensions may be assumed to make a difference: it is a nonsense to say that something is straight in time. So, nothing extended (also) in time falls into the extension of bent. Second option: space and time are uniform in nature, and there is no problem in being straight simpliciter in four dimensions, one of which is temporal. So, there are straight worms and bent worms, just as there are straight slices and bent slices. The only difference is that worms are straight (bent) in a greater number of dimensions (the same difference holds between thick straight objects and flat straight objects).

Both options generate problems. Take the first one. If the candle falls outside the class of plainly straight things inasmuch as it is extended in time, it is non-straight simpliciter. If it is nonstraight simpliciter, however, it is hard to see how it can be straight at times. If 'simpliciter' means anything here indeed, it means 'regardless of anything else', which entails that the candle's being non-straight cannot be affected by the passage of time any more than the Japanese constitution can be affected by the sequence of Swedish kings. So, for every time $t$ at which the candle exists, it must be the case that at $t$ the candle is non-straight. But, if at $t$ the candle is non-straight, then the candle is non-straight-at- $t$. So, if the candle is non-straight simpliciter, at no time can it be straightat-that-time. In a nutshell, being non-straight simpliciter entails being non-straight regardless of the time, which in turn entails being non-straight permanently. Regarding the second option, it is easy to see that it has the same problems, as is evident if one replaces 'non-straight simpliciter' with 'straight simpliciter' and realizes that according to the second option a candle can be straight simpliciter and bent at times, and even straight simpliciter and permanently bent.

Consider the following scheme of argument: 
1) $x$ is $P$ regardless of the time

2) $x$ existed (will exist) at time $t$

3) $x$ was (will be) $P$-at- $t$

This is a very natural scheme of argument, currently occurring in ordinary reasoning and generally assumed to be formally correct (an instance of the scheme in ordinary language is: Anne Windsor is a princess regardless of the time; Anne Windsor existed two years ago; therefore Anne Windsor was a princess two years ago). But the perdurantist can never argue in that way. For she believes that the candle can be non-straight regardless of the time but straight at times and even permanently straight, or straight regardless the time but non-straight at times and even permanently non-straight (as in the case of an ill-made candle). It is easy to see why she believes that. According to her, ' $x$ exists at time $t$ ' means that a temporal part of $x$ exists at $t$, and a straight object may be the sum of non-straight parts, just as a straight object may be the sum of non-straight parts. So far so good, but the fact remains that no translation into 'perdurantese' of arguments of the above form, that are valid in ordinary language, can give us arguments that are valid in perdurantese. This suggests that the 'plain' properties of ordinary persistent objects (those properties that they have simpliciter) do not behave inferentially as the 'plain' properties of worms. ${ }^{19}$ If ordinary objects exist, then they are not worms. And, if everything persistent is a worm, then nothing persistent is an ordinary object. This is an interesting conclusion because the friends of perdurance normally aim to say how ordinary objects - cats, trees and candles - persist. ${ }^{20}$ But what they seem to do is rather something else: to eliminate candles, trees and cats and replace them with things of another sort. Of course this is a possible option for a metaphysical theory. And certainly perdurantism is a coherent metaphysics of persistence. The point is that it is not a theory of how cats, trees and candles persist.

The suspicion is reinforced by the following related point. Take a straight candle moving during a temporal interval (say, today) along a zigzag path. If it is a worm, the worm that it is is non-straight today (for, either the temporal part of the worm that exists today has a fourdimensional zigzag shape, or it is non-straight insofar as it extends in time and nothing temporally extended can be meaningfully said to be straight), but nonetheless it is straight at any moment included in today (since any instantaneous part of the worm is straight). But no candle can fail to be straight today, if it is straight at any moment included in today. Let us call 'temporal distributivity of predication' the principle according to which predication of ordinary objects distributes relative to time just as multiplication distributes relative to addition.

$$
\text { (TDP) } P x \text { at }\left(i^{1}+i^{2}\right) \text { iff }\left[\left(P x \text { at } i^{1}\right) \text { and }\left(P x \text { at } i^{2}\right)\right]
$$

where $i^{1}$ and $i^{2}$ are (non-scattered) temporal intervals, and the variable $x$ ranges over ordinary objects. TDP entails that an object - for example, a candle - is straight at any moment included in a temporal interval, just in case it is straight in the whole interval. The principle seems to be intuitively non-negotiable whenever the property of being $P$ can be had by a persistent object both during a long interval and at a single unextended moment (which is the case with 'straight', but not,

\footnotetext{
${ }^{19}$ This seems to have some bearing on the debate over the purported substantive equivalence of perdurantism and endurantism (see for example Lowe and McCall 2003). For, if what I have argued for is true, then there is no working scheme of translation of 'endurantese' in 'perdurantese'.

${ }^{20}$ There are exceptions. See for example Heller (1990).
} 
for example, with a lingering property like 'drinking') and it seems central in our current way of making inferences about the properties that ordinary objects have at times. But perdurantism entails that the principle fails even in case of properties like straight. The inferential ties between properties had over long times and properties had during shorter sub-times of those longer times are different according to whether those properties are had by ordinary objects or by worms. Inferential ties between sentences of ordinary language fail between their purported translations into perdurantese.

Are stagists and presentists better placed than perdurantists to specify under what conditions, if any, a candle has monadic intrinsic bentness? According to stagists, candles are stages. A stage exists by itself just at one moment, and it is $P$ simpliciter just in case it is $P$ at that very moment. If a candle is now straight, a candle that exists (only) now is straight simpliciter even if it was nonstraight a little while ago (which is the case if and only if it stands in a sort of counterpart relation with another candle-stage that existed a little while ago). According to presentists, all that exists exists now, and at no other time. If a candle is now straight, a candle that exists (only) now is straight simpliciter, and so it is straight simpliciter, even if it was non-straight a little while ago. ${ }^{21}$ Neither stagists nor presentists are in a position to grant the validity of the argument scheme (S) any more than perdurantists. According to stagists, either (2) has only hopelessly false instances, or it means that a temporal counterpart of $\mathrm{x}$ existed (will exist) at $t$, and $x$ normally fails to have the same properties as its temporal counterparts. So, either 2) is false or 3) does not follow from 1) and 2). According to presentists, 3) does not follow from 1) and 2), since the candle might well have now properties it didn't have in the past (or will not have in the future). From both points of view, the candle can be straight simpliciter (and so regardless of the time) and bent at times, which fails to mirror in the technical language of the ontological theory obvious inferential ties between ordinary sentences about persistent objects and their properties. ${ }^{22}$ Again, I suspect that the old familiar entities have been eliminated and replaced with more 'cutting-edge' ones.

\section{Monadic intrinsics properties of enduring things}

So, what about the candle, the property of being bent simpliciter and the eternalist endurantist? Does she have any way to explain under what conditions an enduring thing like a candle has (lacks) a property simpliciter? Here is a suggestion of Peter van Inwagen: 'To say that Descartes had the property of being human is to say that he had that property at any time at which he existed' (van Inwagen 1990, p. 250). The suggestion is made in van Inwagen's adverbialist framework, but this is not important. One might simply say that $x$ is $P$ simpliciter just in case $x$ is permanently $P$ (i.e., $x$ is $P$ at any time at which $x$ exists, existed or will exist); once this is said, it can indifferently be interpreted in adverbialist, relationist, SOFist and even perdurantist terms. What van Inwagen suggests is indeed a sort of analogue of standard supervaluational treatments of vagueness applied to time and predication (in place of supertruth we have truth simpliciter, in place of precisifications we have times, in place of the range of admissible precisifications, we have the temporal limits of

\footnotetext{
${ }^{21}$ In the presentist case, this follows from Prior's well-known 'redundancy theory of the present tense', which is the idea that no grounded distinction can be drawn between present tensed and tenseless sentences (see Prior 1967).

${ }^{22}$ Sider 2000 says that, if candles are stages, it becomes clear how it is that the candle can have both bentness simpliciter and straightness at times (see also Hawley 2000). I think that what stages can but candles cannot do is precisely this. It is this that prevents one to identify candles with stages.
} 
$x$ 's lifespan; and we have the same kind of failure of bivalence cum validity of the excluded middle that is typical of supervaluational logic: if the candle is sometimes straight and sometimes nonstraight, the candle is neither straight simpliciter nor non-straight simpliciter, even though it is straight-or-non-straight simpliciter, for it is straight-or-non-straight permanently). But I suspect that those who claim back monadic intrinsics may have doubts that van Inwagen's suggestion can give them what they want. If monadic properties had simpliciter by objects can be defined in terms of relations with times or the like along supervaluational lines, one may rightly suspect that nothing has been left as a true monadic intrinsic property that objects can have simpliciter.

There may be other difficulties with this suggestion, particularly if one is willing to concede that a predicative connective of weak negation - say nyet - can be introduced such that nyet $(P)$ is true of just those things of which $P$ fails to be true. Since the candle fails to be bent simpliciter, it is nyet(bent) simpliciter, even though it is not nyet(bent) permanently, for it is bent at times. I shall say nothing more about this here, but the general point is that intrinsic monadic properties can be reduced to permanent properties along the above lines only so long as connectives of the nyet sort are not allowed.

If having a property simpliciter is having it regardless of anything else, and particularly regardless of the time, construing bent simpliciter as bent at this or that time (bent now, for example, or being permanently bent) is in some way a betrayal of the very notion of having a property simpliciter. If objects have any properties simpliciter, what these properties are cannot depend on what happens at one or another time. This is the basis of Fine's distinction between sempiternal and eternal truths, where eternal (but not sempieternal) truths 'transcend' the temporal framework. 'An eternal truth', he says, 'will be true regardless of the time, i.e. regardless of how things are at the time, while a sempiternal truth will be true whatever the time, i.e., however things are at the time'. ${ }^{23}$ The distinction between sempiternal and eternal truths parallels a modal distinction between essential and necessary truths. And both distinctions apply equally to kinds of truths and to kinds of properties that objects have. Starting from these intuitions, the eternalist endurantist's suggestion might be: $x$ is $\mathrm{P}$ simpliciter just in case it is $P$ eternally, $P$ essentially, $P$ just in virtue of what $\mathrm{x}$ is. So, Argo is a dog simpliciter because it is a dog in virtue of what it is, and not because of what it happens to it to be at some or each particular moment of its lifespan. And Argo is non-areiform simpliciter because it follows from what it is that it is not aeriform, regardless of how things are arranged at one time or another. But for many things and many properties, what the thing is entails neither that the thing has the property nor that it lacks it. This is the case with all those properties the thing has temporarily, and even with some properties the thing has permanently (one can always be rich, but it does not follow from what she is that she is rich). Some of these properties are monadic. What about them? Does the candle fall into the extension of bent? Outside? Both? Neither?

Here is a suggestion. If something is neither straight in virtue of what it is nor non-straight in virtue of what it is (i.e., neither essentially straight nor essentially non-straight) then it is radically indeterminate whether it is straight simpliciter or not. Being radically indeterminate is not the same as being indeterminate. A sentence is indeterminate just in case it is neither true nor false, whereas it is radically indeterminate just in case it is indeterminate whether it is true and indeterminate whether it is false. In a nutshell, a radically indeterminate sentence is such that not only is there no truth value that the sentence determinately has but even no truth value that the sentence

\footnotetext{
${ }^{23}$ Fine 2005, p. 323.
} 
determinately fails to have, so that ' $p$ is radically indeterminate' entails neither ' $p$ is not true' nor ' $p$ is not false'. ${ }^{24}$ Priest 2006 argues for a dialetheist view of change according to which the candle is both straight and not-straight. A part of Priest's argument is directed toward the idea that the candle is neither straight nor not-straight. According to Priest, that idea entails the dialetheist view because, if the candle is neither straight nor non-straight, then it is straight and not-straight by double negation. Be it as it may, the thesis that it is radically indeterminate whether the candle is straight or not does not entail that it is neither true nor false that the candle is straight, so it does not entail the dialetheist view.

To sum up, straight and bent are monadic intrinsic properties. It is radically indeterminate whether candles have them, but there are entities, for example spatial regions, that determinately have (or fail to have) them. One property can thus be true simpliciter of one entity, false simpliciter of a second entity and radically indeterminate of a third entity, according to what they are, regardless of anything else. If something is $P$ simpliciter, then it is permanently $P$, but the reverse does not hold (being always $P$ is not sufficient for being $P$ simpliciter). Since being $P$ simpliciter entails being $P$ permanently, and being non- $P$ simpliciter entails being non- $P$ permanently (just as $S$ suggests), something can be $P$ at one time and non- $P$ at another only if it is radically indeterminate whether it is $P$ or not: change across time requires radical indeterminacy of timeless properties. What an object is per se underdetermines the properties it has simpliciter. The range of its possible changes is the range of its indeterminacy.

There are a number of reasons for which one might be sceptical about this version of eternalist endurantism. Those who dislike real essences will want to reject the whole account. Those who are suspicious of ontic indeterminacy will want to dismiss it for other reasons. These are issues by far too deep, difficult and ramified to be discussed here. What is clear is that eternalist endurantists can bring back monadic intrinsics properties to enduring things in a number of ways. I have argued that one of these ways is better than any other known alternatives, insofar as it applies to the everyday objects of our commonsensical ontology. In any case, the argument from temporary intrinsics does not constitute an experimentum crucis against eternalist endurantism. The best that perdurantists can reach starting from it is stalemate.

\section{References}

Bradley, F. H. (1897). Appearance and Reality, 2nd edn.. Oxford: Oxford University Press

Eddon, M. (2010). 'Three Arguments from Temporary Intrinsics', Philosophy and Phenomenological Research, 81 (3), 605-619

Fine, K. (2005). 'Necessity and Non-Existence', in K. Fine, Modality and Tense. Philosophical Papers. Oxford: Clarendon Press

Forbes, G. (1987). 'Is there a Problem about Persistence?', Proceedings of the Aristotelian Society, suppl. vol. 61, 137-155

Haslanger, S. (1989). 'Endurance and Temporary Intrinsics', Analysis, 49, pp. 119-25 (2003). 'Persistence through Time'. In M. J. Loux and D. W. Zimmerman (eds.) The Oxford

\footnotetext{
${ }^{24}$ Truth tables for sentential connectives are those defined in Kleene (1952). Kripke (1975) says that Kleene's system is no menace to classical logic. The same holds for radical indeterminacy.
} 
Handbook of Metaphysics: Oxford: Oxford University Press

Hawley, K. (2001), How Things Persist. Oxford: Oxford University Press.

Heller, M. (1990. The Ontology of Physical Objects: Four Dimensional

Hunks of Matter. Cambridge: Cambridge University Press

Hinchliff, M. (1996). 'The Puzzle of Change'. In J. E. Tomberlin (ed.), Philosophical Perspectives, $x$ : Metaphysics. Atascadero CA: Ridgeview.

Humberstone, I. L. (1996). 'Intrinsic/Extrinsic', Synthese, 108, 205-267

Inwagen van, P. (1990). 'Four-Dimensional Objects', Noûs, 24, 245-255

Jackson, F. (1994). 'Metaphysics by Possible Cases', Monist, 77, 103-110

Johnston, M. (1987). 'Is there a Problem about Persistence?', Proceedings of the Aristotelian Society, suppl. vol. 61, 107-135

Kleene, S. C. (1952). Introduction to Metamathematics. North Holland: Amsterdam

Kripke, S. (1975). 'Outline of a Theory of Truth', Journal of Philosophy, 72 (19), 690-716.

Langton, R. and Lewis, D. (1998), 'Defining "Intrinsic"', Philosophy and Phenomenological Research (58), 233-45

Lewis, D. (1986). On the Plurality of Words, Oxford: Blackwell

- $\quad$ (1988). 'Rearrangement of Particles: Reply to Lowe', Analysis, 48: 65-72

- $\quad$ (2002). 'Tensing the Copula', Mind, 111, 1-14

Lowe, E. J. (1988). 'The Problem of Intrinsic Change. Rejoinder to Lewis'. Analysis, 47, 152-154

(1998). The Possibility of Metaphysics. Oxford: Oxford University Press.

Lowe, E. J. and McCall, S. (2003). '3D/4D Equivalence, the Twins Paradox and

Absolute Time'. In Analysis, 63:114-123.

Myro, G. (1987). 'Identity and Time'. In R. Grandy and R. Wagner (eds.), The Philosophical

Grounds of Rationality, (pp. 383-409). New York: Clarendon Press.

Maudlin, T. (2002). Quantum Non-Locality and Relativity. Oxford: Basil Blackwell.

Priest, G. (2006). In Contradiction (2 ${ }^{\text {nd }}$ Edition). Oxford: Oxford University Press

Prior, A. (1967). Past, Present and Future. Oxford: Clarendon Press.

Sider, T. (2000).'The Stage View and Temporary Intrinsics'. Analysis, 60, 84-88.

Wasserman, R. (2006). 'The Problem of Change'. Philosophy Compass, 1 (1), pp. 48-57

Zimmerman, D. (2005) 'The A-Theory of Time, the B-Theory of Time and "Taking Tense

Seriously"', Dialectica, 59 (4), 401-457.

Zimmerman, D. W. (2006). 'Temporary Intrinsics and Presentism'. In S. Haslanger and R.

M. Kurtz, eds., Persistence (pp. 393-423). Cambridge, Mass.: The MIT Press. 